Analele Universităţii de Vest, Timişoara

Seria Matematică - Informatică

LIV, 2, (2016), 47- 58

\title{
Concircular Curvature Tensor of a Semi-symmetric Non-metric Connection on $P$-Sasakian Manifolds
}

Ajit Barman and Gopal Ghosh

\begin{abstract}
The object of the present paper is to study PSasakian manifolds admitting a semi-symmetric non-metric connection whose concircular curvature tensor satisfies certain curvature conditions.
\end{abstract}

AMS Subject Classification (2000). 53C15, 53C25.

Keywords. P-Sasakian manifold, semi-symmetric non-metric connection, concircular curvature tensor, $\xi$-concircularlly flat, $\phi$ concircularly flat, Levi-Civita connection.

\section{Introduction}

In 1924, Friedmann and Schouten [9] introduced the idea of semi-symmetric connection on a differentiable manifold. A linear connection $\bar{\nabla}$ on a differentiable manifold $M$ is said to be a semi-symmetric connection if the torsion tensor $T$ of the connection $\bar{\nabla}$ satisfies $T(X, Y)=u(Y) X-u(X) Y$, where $u$ is a 1 -form and $\xi$ is a vector field defnned by $u(X)=g(X, \xi)$, for all vector fields $X \in \chi(M), \chi(M)$ is the set of all differentiable vector fields on $M$.

In 1932, Hayden [10] introduced the idea of semi-symmetric metric connections on a Riemannian manifold $(M, g)$. A semi-symmetric connection $\widetilde{\nabla}$ is said to be a semi-symmetric metric connection if $\widetilde{\nabla} g=0$ and otherwise 
a semi-symmetric connection $\bar{\nabla}$ is said to be a semi-symmetric non-metric connection if it satisfies the condition $\bar{\nabla} g \neq 0$.

In 1992, Agashe and Chafle [2] studied a semi-symmetric non-metric connection $\bar{\nabla}$, whose torsion tensor $T$ satisfies $T(X, Y)=u(Y) X-u(X) Y$ and $\left(\bar{\nabla}_{X} g\right)(Y, Z)=-u(Y) g(X, Z)-u(Z) g(X, Y)$. They proved that the projective curvature tensor of the manifold with respect to these two connections are equal to each other. In 1992, Barua and Mukhopadhyay [4] studied a type of semi-symmetric connection $\bar{\nabla}$ which satisfies $\left(\bar{\nabla}_{X} g\right)(Y, Z)=$ $2 u(X) g(Y, Z)-u(Y) g(X, Z)-u(Z) g(X, Y)$. Since $\bar{\nabla} g \neq 0$, this is another type of semi-symmetric non-metric connection. However, the authors preferred the name semi-symmetric metric connection.

In 1994, Liang [12] studied another type of semi-symmetric non-metric connection $\bar{\nabla}$ for which we have $\left(\bar{\nabla}_{X} g\right)(Y, Z)=2 u(X) g(Y, Z)$, where $u$ is a non-zero 1-form and he called this a semi-symmetric recurrent metric connection.

The semi-symmetric non-metric connections was further developed by several authors such as Barman and De [6], De and Biswas [8], Liang [12], Singh et al. ([16], [17], [18]), Ozen, Demirbag, Ussal and Yilmaz [13], Smaranda [14], Smaranda and Andonie [15] and many others.

A transformation of a $n$-dimensional Riemannian manifold $M$, which transforms every geodesic circle of $M$ into a geodesic circle, is called a concircular transformation ([11], [20] ). A concircular transformation is always a conformal transformation [11]. Here geodesic circle means a curve in $M$ whose first curvature is constant and whose second curvature is identically zero. Thus the geometry of concircular transformations, i.e., the concircular geometry, is generalization of inversive geometry in the sense that the change of metric is more general than that induced by a circle preserving diffeomorphism (see also [7]). An interesting invariant of a concircular transformation is the concircular curvature tensor $\mathbf{C}$ with respect to the Levi-Civita connection. It is defnned by ([20], [21])

$$
\mathbf{C}(X, Y) Z=R(X, Y) Z-\frac{r}{n(n-1)}[g(Y, Z) X-g(X, Z) Y],
$$

where $X, Y, Z, \in \chi(M), R$ and $r$ are curvature tensor and the scalar curvature with respect to the Levi-Civita connection respectively. Riemannian manifolds with vanishing concircular curvature tensor are of constant curvature. Thus, the concircular curvature tensor is a measure of the failure of a Riemannian manifold to be of constant curvature.

In this paper we study concircular curvature tensor on P-Sasakian manifolds with respect to a type of semi-symmetric non-metric connection due to Agashe and Chafle [2]. The paper is organized as follows: After introduction 
in section 2, we give a brief account of the P-Sasakian manifolds. In section 3, we obtain the expression the semi-symmetric non-metric connection on P-Sasakian manifolds. Section 4 is devoted to study $\xi$-concircularly flat P-Sasakian manifolds admitting the semi-symmetric non-metric connection. Section 5 deals with $\phi$-concircularly flat P-Sasakian manifolds with respect to the semi-symmetric non-metric connection. In the next section we have classified P-Sasakian manifolds which satisfy $\overline{\mathbf{C}} \cdot \bar{S}=0$ with respect to the semi-symmetric non-metric connection, where $\bar{S}$ and $\overline{\mathbf{C}}$ denote the Ricci tensor and the concircular curvature tensor with respect to the semi-symmetric non-metric connection respectively. Finally, we construct an example of a 3dimensional P-Sasakian manifold admitting the semi-symmetric non-metric connection to support the results obtained in Section 4 and Section 5 respectively.

\section{P-Sasakian manifolds}

An $n$-dimensional differentiable manifold $M$ is said to admit an almost paracontact Riemannian structure $(\phi, \xi, \eta, g)$, where $\phi$ is a $(1,1)$ - tensor field, $\xi$ is a vector field, $\eta$ is a 1 -form and $g$ is a Riemannian metric on $M$ such that

$$
\begin{gathered}
\phi \xi=0, \quad \eta(\phi X)=0, \quad \eta(\xi)=1, \quad g(X, \xi)=\eta(X) \\
\phi^{2}(X)=X-\eta(X) \xi \\
g(\phi X, \phi Y)=g(X, Y)-\eta(X) \eta(Y) \\
\left(\nabla_{X} \eta\right) Y=g(X, \phi Y)=\left(\nabla_{Y} \eta\right) X
\end{gathered}
$$

for any vector fields $X, Y$ on $M$.

If moreover, $(\phi, \xi, \eta, g)$ satisfy the relations

$$
\begin{gathered}
\nabla_{X} \xi=\phi X \\
d \eta=0,\left(\nabla_{X} \phi\right) Y=-g(X, Y) \xi-\eta(Y) X+2 \eta(X) \eta(Y) \xi
\end{gathered}
$$

then $M$ is called para-Sasakian manifold or briefly, P-Sasakian manifold. It is known ([1], [19]) that in a P-Sasakian manifold, the following relations hold:

$$
\eta(R(X, Y) Z)=g(X, Z) \eta(Y)-g(Y, Z) \eta(X)
$$




$$
\begin{gathered}
R(\xi, X) Y=\eta(Y) X-g(X, Y) \xi \\
R(X, Y) \xi=\eta(X) Y-\eta(Y) X \\
S(X, \xi)=-(n-1) \eta(X), \\
S(\phi X, \phi Y)=S(X, Y)+(n-1) \eta(X) \eta(Y),
\end{gathered}
$$

where $S$ denotes the Ricci tensor of the Levi-Civita connection $\nabla$.

\section{Semi-symmetric non-metric connection on P-Sasakian manifolds}

This section deals with a type of semi-symmetric non-metric connection on a P-Sasakian manifold. We consider a type of semi-symmetric non-metric connection which was introduced by Agashe and Cheffle [2]. In a P-Sasakian manifold a semi-symmetric non-metric connection is defnned by $T(X, Y)=$ $\eta(Y) X-\eta(X) Y$, with $\xi$ as the associated vector field i.e. $g(X, \xi)=\eta(X)$.

A relation between semi-symmetric non-metric connection $\bar{\nabla}$ and the Levi-Civita connection $\nabla$ have been obtained by Agashe and Cheffle [2] and is given by

$$
\bar{\nabla}_{X} Y=\nabla_{X} Y+\eta(Y) X
$$

Using (3.1), the torsion tensor $T$ of $M$ with respect to the connection $\bar{\nabla}$ is given by

$$
T(X, Y)=\bar{\nabla}_{X} Y-\bar{\nabla}_{Y} X-[X, Y]=\eta(Y) X-\eta(X) Y .
$$

Hence a relation satisfying (3.2) is called a semi-symmetric connection. Further using (3.1), we have

$$
\left(\bar{\nabla}_{X} g\right)(Y, Z)=-u(Y) g(X, Z)-u(Z) g(X, Y) \neq 0 .
$$

$\bar{\nabla}$ defnned by (3.1) satisfying (3.2) and (3.3) is a type of semi-symmetric non-metric connection.

Now, we obtain the expressions of the curvature tensor, Ricci tensor and scalar curvature of $M$ with respect to the semi-symmetric non-metric connection defnned by (3.1). 
Analogous to the defnnitions of the curvature tensor $R$ of $M$ with respect to the Levi-Civita connection $\nabla$, we defnne the curvature tensor $\bar{R}$ of $M$ with respect to the semi-symmetric non-metric connection $\bar{\nabla}$ given by $\bar{R}(X, Y) Z=\bar{\nabla}_{X} \bar{\nabla}_{Y} Z-\bar{\nabla}_{Y} \bar{\nabla}_{X} Z-\bar{\nabla}_{[X, Y]} Z$, where $X, Y, Z \in \chi(M)$, the set of all differentiable vector fields on $M$. Then $\bar{R}$ and $R$ are related by [5]

$$
\begin{array}{r}
\bar{R}(X, Y) Z=R(X, Y) Z+g(X, \phi Z) Y-\eta(X) \eta(Z) Y-g(Y, \phi Z) X \\
+\eta(Y) \eta(Z) X .
\end{array}
$$

The Ricci tensors $\bar{S}$ and $S$ with respect to the semi-symmetric non-metric connection and the Levi-Civita connection respectively are related by [5]

$$
\bar{S}(Y, Z)=S(Y, Z)-(n-1) g(Y, \phi Z)+(n-1) \eta(Y) \eta(Z)
$$

and also [5]

$$
\bar{S}(Y, \xi)=0 .
$$

Again $\bar{r}$ and $r$ are related by [5]

$$
\bar{r}=r-(n-1) \beta+n-1=r-(n-1)(\beta-1),
$$

where $\bar{r}$ and $r$ are the scalar curvature with respect to the semi-symmetric non-metric connection and the Levi-Civita connection respectively and $\beta=$ trace $\phi$.

\section{$4 \quad \xi$-concircularly flat P-Sasakian manifolds with re- spect to the semi-symmetric non-metric connection}

The concircular curvature tensor $\overline{\mathbf{C}}$ with respect to the semi-symmetric nonmetric connection is given by

$$
\overline{\mathbf{C}}(X, Y) Z=\bar{R}(X, Y) Z-\frac{\bar{r}}{n(n-1)}[g(Y, Z) X-g(X, Z) Y] .
$$

Definition 4.1. A P-Sasakian manifold with respect to the semi-symmetric non-metric connection $\bar{\nabla}$ is said to be $\xi$-concircularly flat if $\overline{\boldsymbol{C}}(X, Y) \xi=0$.

In view of (3.4), (3.7) and (4.1) yields

$$
\begin{array}{r}
\overline{\mathbf{C}}(X, Y) Z=R(X, Y) Z+g(X, \phi Z) Y-\eta(X) \eta(Z) Y-g(Y, \phi Z) X \\
+\eta(Y) \eta(Z) X-\frac{r-(n-1)(\beta-1)}{n(n-1)}[g(Y, Z) X-g(X, Z) Y],
\end{array}
$$


where $\beta=$ trace $\phi$.

Taking $Z=\xi$ in (4.2) and using (2.1) and (2.2), it follows that

$$
\overline{\mathbf{C}}(X, Y) \xi=\frac{r-(n-1)(\beta-1)}{n(n-1)} R(X, Y) \xi .
$$

Thus, we have the following:

Theorem 4.1. A P-Sasakian manifold admitting a semi-symmetric nonmetric connection is $\xi$-concircularly flat if the scalar curvature tensor with respect to the Levi-Civita connection is equal to $(n-1)(\beta-1)$.

\section{$5 \phi$-concircularly flat P-Sasakian manifolds admitting a semi-symmetric non-metric connection}

Definition 5.1. A P-Sasakian manifold is said to be $\phi$-concircularly flat with respect to the semi-symmetric non-metric connection $\bar{\nabla}$ if

$$
g(\overline{\boldsymbol{C}}(\phi X, \phi Y) \phi Z, \phi W)=0,
$$

where $X, Y, Z, W \in \chi(M)$.

Taking the inner product of (4.1) with $W$, we have

$$
\begin{array}{r}
{ }^{\prime} \mathbf{C}(X, Y, Z, W)={ }^{\prime} \bar{R}(X, Y, Z, W)-\frac{\bar{r}}{n(n-1)}[g(Y, Z) g(X, W) \\
-g(X, Z) g(Y, W)],
\end{array}
$$

where ${ }^{\prime} \overline{\mathbf{C}}$ and $\overline{\mathbf{C}}$ are the concircular curvature tensor of type $(0,4)$ and $(1,3)$ with respect to the semi-symmetric non-metric connection, respectively, and ${ }^{\prime} \overline{\mathbf{C}}(X, Y, Z, W)=g(\overline{\mathbf{C}}(X, Y) Z, W)$ and ${ }^{\prime} \bar{R}(X, Y, Z, W)=g(\bar{R}(X, Y) Z, W)$.

Computing (5.2) in $\phi X, \phi Y, \phi Z, \phi W$, we get

$$
\begin{array}{r}
{ }^{\prime} \mathbf{C}(\phi X, \phi Y, \phi Z, \phi W)={ }^{\prime} \bar{R}(\phi X, \phi Y, \phi Z, \phi W)-\frac{\bar{r}}{n(n-1)}[g(\phi Y, \phi Z) g(\phi X, \phi W) \\
-g(\phi X, \phi Z) g(\phi Y, \phi W)](5.3)
\end{array}
$$

Combining (5.1) and (5.3), it follows that

$$
\begin{array}{r}
{ }^{\prime} \bar{R}(\phi X, \phi Y, \phi Z, \phi W)-\frac{\bar{r}}{n(n-1)}[g(\phi Y, \phi Z) g(\phi X, \phi W) \\
-g(\phi X, \phi Z) g(\phi Y, \phi W)]=0 .
\end{array}
$$


Let $\left\{e_{1}, \ldots, e_{n-1}, \xi\right\}$ be a local orthonormal basis of vector fields in $M$, then $\left\{\phi e_{1}, \ldots, \phi e_{n-1}, \xi\right\}$ is also a local orthonormal basis. Putting $X=W=e_{i}$ in (5.4) and summing over $i=1$ to $n-1$, we obtain

$$
\begin{array}{r}
\sum_{i=1}^{n-1} g\left(\bar{R}\left(\phi e_{i}, \phi Y,\right) \phi Z, \phi e_{i}\right)-\frac{\bar{r}}{n(n-1)} \sum_{i=1}^{n-1}\left[g(\phi Y, \phi Z) g\left(\phi e_{i}, \phi e_{i}\right)\right. \\
\left.-g\left(\phi e_{i}, \phi Z\right) g\left(\phi Y, \phi e_{i}\right)\right]=0 .
\end{array}
$$

So the equation (5.5) turns into

$$
\bar{S}(\phi Y, \phi Z)-\frac{\bar{r}(n-2)}{n(n-1)} g(\phi Y, \phi Z)=0 .
$$

Using (2.1), (2.2), (3.5) and (3.7) in (5.6), implies that

$$
\begin{array}{r}
S(\phi Y, \phi Z)-(n-1) g(\phi Y, Z)-\frac{[r-(n-1)(\beta-1)](n-2)}{n(n-1)} \\
g(\phi Y, \phi Z)=0 .
\end{array}
$$

By making use of $(2.3),(2.11)$ and (5.7), it is obvious that

$$
\begin{gathered}
S(Y, Z)+(n-1) \eta(Y) \eta(Z)-(n-1) g(\phi Y, Z)- \\
\frac{[r-(n-1)(\beta-1)](n-2)}{n(n-1)}[g(Y, Z) \\
-\eta(Y) \eta(Z)]=0 .
\end{gathered}
$$

Then contracting the last equation over $Y$ and $Z$ and using (2.1), we obtain

$$
r=(n-1)(\beta-1) \text {. }
$$

The above discussion helps us to state the following:

Theorem 5.1. A P-Sasakian manifold is $\phi$-concircularly flat with respect to the semi-symmetric non-metric connection, then the scalar curvature tensor of the Levi-Civita connection of $M$ is equal to $(n-1)(\beta-1)$, where $\beta=$ trace of $\phi$.

\section{$6 \quad$ P-Sasakian manifolds satisfying $\overline{\mathbf{C}} \cdot \bar{S}=0$ with respect to a semi-symmetric non-metric connection}

In this section, we consider P-Sasakian manifolds with respect to a semisymmetric non-metric connection $\bar{\nabla}$ satisfying $\overline{\mathbf{C}} \cdot \bar{S}=0$. Then

$$
(\bar{C}(X, Y) \cdot \bar{S})(U, V)=0
$$


So,

$$
\bar{S}(\bar{C}(X, Y) U, V)+\bar{S}(U, \bar{C}(X, Y) V)=0 .
$$

Taking $V=\xi$ in (6.1) and using (3.6), it follows that

$$
\bar{S}(U, \bar{C}(X, Y) \xi)=0 .
$$

Applying (2.1), (2.9) and (4.2) in (6.2), we see that

$$
\frac{r-(n-1)(\beta-1)}{n(n-1)}[\eta(X) \bar{S}(U, Y)+\eta(Y) \bar{S}(U, X)]=0 .
$$

Taking $X=\xi$ in (6.3) and using (3.6) and (2.1), we have

$$
\frac{r-(n-1)(\beta-1)}{n(n-1)} \bar{S}(U, Y)=0 .
$$

Using (3.5) in (6.4), we have

$$
\frac{r-(n-1)(\beta-1)}{n(n-1)}[S(U, Y)-(n-1) g(U, \phi Y)+(n-1) \eta(Y) \eta(U)]=0 .
$$

Then contracting the last equation over $Y$ and $U$ and using (2.1), we obtain

$$
\frac{[r-(n-1)(\beta-1)]^{2}}{n(n-1)}=0,
$$

which implies that

$$
r=(n-1)(\beta-1) .
$$

This leads to the following:

Theorem 6.1. If a P-Sasakian manifolds satisfying $\overline{\boldsymbol{C}} \cdot \bar{S}=0$ with respect to the semi-symmetric non-metric connection, then the scalar curvature tensor of the Levi-Civita connection of $M$ is equal to $(n-1)(\beta-1)$, where $\beta$ is the trace of $\phi$.

\section{Example}

In this section we construct an example on P-Sasakian manifold with respect to the semi-symmetric non-metric connection $\bar{\nabla}$ which verify the results of Section 4 and Section 5. 
We consider the 3-dimensional manifold $\left\{(x, y, z) \in \mathbb{R}^{3}\right\}$, where $(x, y, z)$ are the standard coordinates in $\mathbb{R}^{3}$.

We choose the vector fields

$$
e_{1}=z \frac{\partial}{\partial x}, e_{2}=z \frac{\partial}{\partial y}, e_{3}=-z \frac{\partial}{\partial z}
$$

which are linearly independent at each point of $M$.

Let $g$ be the Riemannian metric defnned by

$$
g\left(e_{i}, e_{j}\right)= \begin{cases}1 & \text { if } \quad i=j \\ 0 & \text { if } \quad i \neq j ; i, j=1,2,3 .\end{cases}
$$

Let $\eta$ be the 1-form defnned by

$$
\eta(Z)=g\left(Z, e_{3}\right)
$$

for any $Z \in \chi(M)$.

Let $\phi$ be the $(1,1)$-tensor field defnned by

$$
\phi\left(e_{1}\right)=e_{1}, \phi\left(e_{2}\right)=e_{2}, \phi\left(e_{3}\right)=0 .
$$

Using the linearity of $\phi$ and $g$, we have

$$
\eta\left(e_{3}\right)=1, \phi^{2} Z=Z-\eta(Z) e_{3}
$$

and

$$
g(\phi Z, \phi U)=g(Z, U)-\eta(Z) \eta(U),
$$

for any vector fields $Z, U \in \chi(M)$. Thus for $e_{3}=\xi$, the structure $(\phi, \xi, \eta, g)$ defnnes an almost paracontact metric structure on $M$.

Then we have

$$
\left[e_{1}, e_{2}\right]=0,\left[e_{1}, e_{3}\right]=e_{1},\left[e_{2}, e_{3}\right]=e_{2}
$$

The Levi-Civita connection $\nabla$ of the metric tensor $g$ is given by Koszul's formula which is given by

$$
\begin{aligned}
2 g\left(\nabla_{X} Y, Z\right)= & X g(Y, Z)+Y g(Z, X)-Z g(X, Y) \\
& -g(X,[Y, Z])-g(Y,[X, Z])+g(Z,[X, Y]) .
\end{aligned}
$$

Using Koszul's formula, we get the following:

$$
\begin{aligned}
& \nabla_{e_{1}} e_{1}=-e_{3}, \nabla_{e_{1}} e_{2}=0, \nabla_{e_{1}} e_{3}=e_{1} \\
& \nabla_{e_{2}} e_{1}=0, \nabla_{e_{2}} e_{2}=-e_{3}, \nabla_{e_{2}} e_{3}=e_{2} \\
& \nabla_{e_{3}} e_{1}=0, \nabla_{e_{3}} e_{2}=0, \nabla_{e_{3}} e_{3}=0
\end{aligned}
$$

In view of the above relations, we see that 
$\nabla_{X} \xi=\phi X,\left(\nabla_{X} \phi\right) Y=-g(X, Y) \xi-\eta(Y) X+2 \eta(X) \eta(Y) \xi$, for all $e_{3}=\xi$.

Therefore the manifold $M$ is P-Sasakian with the structure $(\phi, \xi, \eta, g)$. Using (3.1) in the above equations, we obtain

$$
\begin{aligned}
& \bar{\nabla}_{e_{1}} e_{1}=-e_{3}, \bar{\nabla}_{e_{1}} e_{2}=0, \bar{\nabla}_{e_{1}} e_{3}=2 e_{1}, \\
& \bar{\nabla}_{e_{2}} e_{1}=0, \bar{\nabla}_{e_{2}} e_{2}=-e_{3}, \bar{\nabla}_{e_{2}} e_{3}=2 e_{2}, \\
& \bar{\nabla}_{e_{3}} e_{1}=0, \bar{\nabla}_{e_{3}} e_{2}=0, \bar{\nabla}_{e_{3}} e_{3}=e_{3} .
\end{aligned}
$$

Now, we can easily obtain the non-zero components of the curvature tensors as follows:

$$
\begin{aligned}
& R\left(e_{1}, e_{2}\right) e_{2}=-e_{1}, R\left(e_{1}, e_{3}\right) e_{3}=-e_{1}, R\left(e_{2}, e_{1}\right) e_{1}=-e_{2}, \\
& R\left(e_{2}, e_{3}\right) e_{3}=-e_{2}, R\left(e_{3}, e_{1}\right) e_{1}=-e_{3}, R\left(e_{3}, e_{2}\right) e_{2}=-e_{3} .
\end{aligned}
$$

and

$$
\begin{aligned}
& \bar{R}\left(e_{1}, e_{2}\right) e_{2}=-2 e_{1}, \bar{R}\left(e_{2}, e_{1}\right) e_{1}=-2 e_{2}, \\
& \bar{R}\left(e_{3}, e_{1}\right) e_{1}=-2 e_{3}, \bar{R}\left(e_{3}, e_{2}\right) e_{2}=-2 e_{3} .
\end{aligned}
$$

With the help of the above results we find the Ricci tensors as follows:

$$
S\left(e_{1}, e_{1}\right)=S\left(e_{2}, e_{2}\right)=S\left(e_{3}, e_{3}\right)=-2
$$

and

$$
\bar{S}\left(e_{1}, e_{1}\right)=\bar{S}\left(e_{2}, e_{2}\right)-4, \bar{S}\left(e_{3}, e_{3}\right)=0 .
$$

Also it follows that the scalar curvature with respect to the Levi-Civita connection and the semi-symmetric non-metric connection are $r=-6$ and $\bar{r}=-8$, respectively.

Let $X, Y, Z$ and $W$ be any four vector fields given by $X=a_{1} e_{1}+a_{2} e_{2}+a_{3} e_{3}, Y=b_{1} e_{1}+b_{2} e_{2}+b_{3} e_{3}, Z=c_{1} e_{1}+c_{2} e_{2}+c_{3} e_{3}$ and $W=d_{1} e_{1}+d_{2} e_{2}+d_{3} e_{3}$, where $a_{i}, b_{i}, c_{i}, d_{i}$, for all $i=1,2,3$, are non-zero real numbers.

Using the above curvature tensors and the scalar curvature of the semisymmetric non-metric connection, we obtain

$$
\overline{\mathbf{C}}(X, Y) \xi=\frac{4}{3}\left[\left(a_{1} b_{3}-a_{3} b_{1}\right) e_{1}+\left(a_{2} b_{3}-a_{3} b_{2}\right) e_{2}\right]
$$


and

$$
\overline{\mathbf{C}}(\phi X, \phi Y, \phi Z, \phi W)=-2\left(a_{1} b_{2} c_{2} d_{1}+a_{2} b_{1} c_{1} d_{2}-\frac{4}{3}\right) .
$$

From the above two relations, we see that the P-Sasakian manifold $M$ will be $\xi$-concircularly flat and $\phi$-concircularly flat with respect to the semisymmetric non-metric connections if $\frac{a_{1}}{b_{1}}=\frac{a_{2}}{b_{2}}=\frac{a_{3}}{b_{3}}$ and $a_{1} b_{2} c_{2} d_{1}+a_{2} b_{1} c_{1} d_{2}=\frac{4}{3}$ which verify the results of Section 4 and Section 5 , respectively.

\section{Acknowledgement}

The authors are thankful to the referee for his/her valuable suggestions for the improvement of the paper.

\section{References}

[1] T. Adati and K. Matsumoto, On conformally recurrent and conformally symmetric P-Sasakian manifolds, TRU Math., 13, (1977), 25-32

[2] N. S. Agashe and M. R. Chafle, A semi-symmetric non-metric connection on a Riemannian Manifold, Indian J. Pure Appl. Math., 23, (1992), 399-409

[3] O. C. Andonie, On semi-symmetric non-metric connection on a Riemannian manifold, Ann. Fac. Sci. De Kinshasa, Zaire Sect. Math. Phys., 2, (1976)

[4] B. Barua and S. Mukhopadhyay, A sequence of semi-symmetric connections on a Riemannian manifold, Proceedings of seventh national seminar on Finsler, Lagrange and Hamiltonian spaces, Brasov, Romania., (1992)

[5] A. Barman, Semi-symmetric non-metric connection in a P-Sasakian manifold, Novi Sad J. Math., 43, (2013), 117-124

[6] A. Barman and U. C. De, Semi-symmetric non-metric connections on Kenmotsu manifolds, Romanian J. Math. and Comp. Sci., 5, (2014), 13-24

[7] D. E. Blair, Inversion theory and conformal mapping, Student Mathematical Library 9, American Mathematical Society, (2000)

[8] U. C. De and S. C. Biswas, On a type of semi-symmetric non-metric connection on a Riemannian manifold, Ganita, 48, (1997), 91-94

[9] A. Friedman and J. A. Schouten, $\ddot{U}$ ber die Geometric der halbsymmetrischen Übertragung, Math., Zeitschr., 21, (1924), 211-223

[10] H. A. Hayden, Subspaces of space with torsion, Proc. London Math. Soc., 34, (1932), 27-50

[11] W. Kuhnel, Conformal transformations between Einstein spaces, Conformal geometry (Bonn, 1985/1986 ), Aspects Math., E12, Vieweg, Braunschweig, (1988), 105-146 
[12] Y. Liang, On semi-symmetric recurrent-metric connection, Tensor, N. S., 55, (1994), $107-112$

[13] Z. F. Ozen, S. A. Demirbag, S. A. Uysal, and H. B. Yilmaz, Some vector fields on a Riemannian manifold with semi-symmetric metric connection, Bull. Iranian Math. Soc., 38, (2012), 479-490

[14] D. Smaranda, Pseudo Riemannian recurrent manifolds with almost constant curvature, The XVIII Int. conf. on Geometry and Topology (Oradea 1989), pp 88-2, Univ. "Babes Bolyai" Cluj-Napoca, 2, (1988)

[15] D. Smaranda and O. C. Andonie, On semi-symmetric connections, Ann. Fac. Sci. Univ. Nat. Zaire (Kinshasa), Sec. Math.-Phys., 2, (1976), 266-270

[16] R. N. Singh, On a product semi-symmetric non-metric connection in a locally decomposable Riemannian manifold, International Math. Forum, 6, (2011), 1893-1902

[17] R. N. Singh and G. Pandey, On the $W_{2}$-curvature tensor of the semi-symmetric non-metric connection in a Kenmotsu manifold, Navi Sad J. Math., 43, (2013), 91105

[18] R. N. Singh and M. K. Pandey, On semi-symmetric non-metric connection I, Ganita, 58, (2007), 47-59

[19] I. Sato, On a structure similar to the almost contact structure, Tensor, (N.S.), 30, (1976), 219-224

[20] K. Yano, Concircular geometry I. Concircular transformations, Proc. Imp. Acad. Tokyo, 16, (1940), 195-200

[21] K. Yano and S. Bochner, Curvature and Betti numbers, Annals of Mathematics studies, 32 Princeton University Press, (1953)

Ajit Barman

Department of Mathematics, Kabi Nazrul Mahavidyalaya, P.O.:-Sonamura-799181,P.S.-Sonamura,Dist- Sepahijala, Tripura, India.

E-mail: ajitbarmanaw@yahoo.in

Gopal Ghosh

Department of Pure Mathematics, University of Calcutta, 35, Ballygunge Circular Road, Kolkata-700019, WB, India.

E-mail: ghoshgopal.pmath@gmail.com

Received: 7.08.2015

Accepted: 26.10.2016

Revised: 27.07.2016 